with the same spongework of very delicate connective tissue as the drainage area. Here and there also are some spots of pigment.'

The walls of the track are in part lined by very definite endothelium (Figs. 9 and 10), while in others the sclera is bare, and it is a very remarkable feature that where the sclera is bare it has apparently undergone no change since the day the incision was made some twelve years ago.

In many of the sections a portion of Descemet's membrane is seen (Fig. 9). This is no doubt due to the fact that Descemet's membrane tends to curl forwards when cut.

\title{
Comment
}

An endothelial lining to filtering scars is usually denied but there can be no doubt of its presence here. The fact that the sclera has shown no signs of healing appears to bear out Thomson Henderson's theory that tissues bathed by the aqueous are not stimulated to show the usual signs of repair.

\section{THE FLAP SCLEROTOMY IN THE TREATMENT OF GLAUCOMA*}

\author{
BY
}

\author{
W. M. DE C. BOXILL \\ LATE R.M.O.,.ROYAL WESTMINSTER \\ OPHTHALMIC HOSPITAL
}

Since the introduction of a similar operation by the late Colonel Herbert in 1907 , but based on an erroneous hypothesis, the technique of the flap-sclerotomy appears to have been considerably varied by those few who, in the past, have used it in the treatment of chronic primary glaucoma. The technique, which will later be described, has arisen through the work of Sir Richard Cruise at the Royal Westminster Ophthalmic Hospital during his long and successful association with that hospital, and it is through the efforts of this great master of the simple extraction operation for cataract that an even simpler method of permanent decompression of the hypertensive eye has been evolved.

In the past this operation has mostly been confined to cases of chronic glaucoma particularly the primary ones. Recently it has been performed with success on acute congestive hypertensive eyes which did not respond to medical treatment. The technique in both

- Received for publication, January 2, 1947. 
cases being the same with the pre-operative treatment varied accordingly.

The factors in favour of this operation apply equally to both acute and chronic cases which require operative treatment. They are as follows :-

(a) This is the least traumatic of all the operations for ocular decompression. No tissue is excised. An iridectomy is unnecessary and therefore avoided, hence there is no haemorrhage into the anterior chamber with the subsequent passage of blood admixed with aqueous into the sclero-corneal wound thereby facilitating its blockage and early closure;

(b). Slow decompression of the anterior chamber;

(c) The permanent incisions into the angle of the anterior chamber are covered by a flap of conjunctiva and Tenon's capsule and therefore protected from the conjunctival sac. The advantage of this in the acute case with a potentially dirty conjunctival sac is clear ;

(d) It is an easy operation to perform.

The pre-operative treatment is slightly different in the two types of case. In the primary acute eye, operative treatment is generally adopted when the pupil and tension do not respond to intensive treatment with miotics, heat, etc. It seems likely that here the corneal oedema is so great that miotics are unable to pass through the oedematous barrier in sufficient concentration to be effective. At this stage, when it has been decided to adopt surgical measures, a retro-bulbar injection of 1 c.c. of 3 per cent. novocaine is given through the lower lid with a $1 \frac{1}{4}$ " needle and the commencement of the operation delayed until the tension is lowered sufficiently for the corneal oedema to clear and the pupil to respond to miotics which are now able to pass through the cornea in effective concentration, also the conjunctiva by now is less congested and the slight bleeding which is subsequently encountered at operation is easily controlled. Furthermore, as the cessation of pain rapidly follows the retro-bulbar injection, the apprehension of the patient is lessened and the operation can proceed under conditions approximating those prevailing with a cold case.

If 1 e.c. of 3 per cent., novocaine is not sufficient, which is rare, the injection can be repeated giving $\frac{1}{2}$ c.c. at a time until the condition of the eye is such that operative procedure can be undertaken under ideal conditions.

As the conjunctival sac is potentially dirty, just prior to the operation it is washed out with a sterile warm solution of normal saline and guttae penicillin 500-1,000 units per c.c. in normal saline instilled. Penicillin drops can also be used during the period of medical treatment with miotics, etc.

With regard to the giving of a retro-bulbar injection to an eye 
whose extra-ocular tissue is very congested, there are some who would say that the dangers of intra-orbital haemorrhage following this injection are greatly increased when it is given in this type of case, however, assurance has been obtained by no less an authority than Mr. Eugene Wolff of the Royal. Westminster Ophthalmic Hospital, that there is no increase in the occurrence of intra-orbital haemorrhage providing the injection is given with a needle not more than $1 \frac{1}{4}$ in. in length and in practice, so far, this dreaded complication has not arisen.

In the case of chronic primary glaucoma, the pre-operative requirements are both few and simple. Eserine $\frac{1}{2}-1$ per cent. is frequently instilled into the conjunctival sac $1-1 \frac{1}{2}$ hours before the operation so that the pupil is as small as possible and well under the influence of this drug, also, guttae pantocaine 1 per cent. at frequent intervals $\frac{1}{2}-\frac{3}{4}$ hour before the operation to ensure good anaesthesia.

In the theatre $\frac{1}{2}-\frac{3}{4}$ c.c. of 3 per cent. novocaine is injected subconjunctivally in and around the tendon of insertion of the superior rectus muscle. The advantages of this injection are :

(a) Paralysis of the superior rectus muscle to prevent the patient looking upwards during the operation.

(b) Increase of the anaesthesia of the conjunctiva and Tenon's capsule in the immediate area of operation.

This injection is not usually required when dealing with an acute case if the retro-bulbar injection has been given into the muscle cone around the ciliary ganglion and time enough has been allowed to elapse for its anaesthetic and paralytic action to have full effect. However, $\frac{1}{4}-\frac{1}{2}$ c.c. of 3 per cent. novocaine with $1 \mathrm{minim}$ of adrenalin $1 / 1,000$ added may be injected sub-conjunctivally around the insertion of the superior rectus muscle in those acute cases where the conjunctival vessels are excessively congested, in order to reduce bleeding when the conjunctival flap is being cut.

The technique of the operation is as follows :

A small snip is made into the conjunctiva and Tenon's capsule about $6 \mathrm{~mm}$. directly above 12 o'clock on the cornea, this snip is then extended medially and laterally and for an equal distance on both sides of the original snip so that an incision is obtained which is roughly $1-1 \frac{1}{2} \mathrm{cms}$. in length and is parallel with the limbus. This flap composed of the above two structures is then turned downwards and gradually dissected off the sclera using non-toothed forceps for fixation and blunt nosed conjunctival scissors for the dissection until about $2 \mathrm{~mm}$. from the sclero-corneal junction, when sharp-pointed scissors are substituted to complete the dissection. The actual snips made in this region should be as near to the sclera as possible; if not the conjunctiva may be button-holed, for in this region the conjunctiva, Tenon's capsule and episcleral tissue are 
fused and therefore not as easily raised from the sclera as has been previously encountered. During this part of the dissection the sclero-corneal junction is reached and recognised by the sharp demarcation of the grey tissue of the cornea and of the white sclera; the junction where these two meet may be referred to as the "grey line."

When the "grey line" is reached, which usually first appears in the centre of the dissection, the dissection is then carried laterally and medially with sharp-pointed scissors so that the "grey line" is exposed for about 4-5 mm. As soon as this step has been completed, a Tooke's corneal splitter is used to carry the dissection forwards in the same plane in order to separate the corneal tissues the whole length of the exposed "grey line" and for a distance of $1 \frac{1}{2} \mathrm{~mm}$. in front of it ; the raised portion of corneal tissue remaining in continuity with the previously raised conjunctival flap above and the cornea below.

The above dissections having been completed, oozing episcleral and conjunctival vessels should be dealt with in order to provide a clean dry field in preparation for the incisions now to be made into the scleral and corneal tissues. The conjunctival flap is now turned downwards on to the cornea with its conjunctival surface in apposition with the anterior surface of the cornea : the assistant steadying the flap with a small swab soaked in normal saline and held between the tips of non-toothed forceps; the swab being applied to the Tenon surface of the flap, traction being gently applied in a downward direction, but without exerting pressure on the eye.

There is now an area of the outer surface of the angle of the anterior chamber exposed for the permanent incisions which are to be made in this situation, but before commencing these incisions, it is essential that perfect fixation of the eye is assured. Of the many methods of fixation, the aim of which is to render the eye immobile without exerting external pressure upon it, the one which has been found to answer these requirements is by the use of the scleral hook, whereby the superficial scleral fibres are picked up by passing a small sharp pointed hook beneath them. The hook most commonly used in this operation is the Cruise scleral hook (John Weiss and Co.) which has been specially designed for this operation.

The next step in the operation is the insertion of the scleral hook, the place of insertion being along the "grey line," the points of entrance and exit of the hook being approximately on either side of, and equidistant from, 12 o'clock on the cornea, the distance between these points being about $1-1 \frac{1}{2} \mathrm{~mm}$. With the scleral hook in situ and held by the free hand of the operator, an incision is now made with a small scalpel or Graefe knife into the sclera $5 \mathrm{~mm}$. long and $1 \mathrm{~mm}$. from and parallel to the " grey line," the mid-point of the incision being approximately opposite 12 o'clock on the cornea. 
Since the sclera extends for a distance of $2 \mathrm{~mm}$. from the anterior extremity of the ciliary body to the sclero-corneal junction in this upper portion of the anterior chamber, the base incision just mentioned is therefore placed $1 \mathrm{~mm}$. in front of the root of the iris, so that neither this tissue nor the ciliary body is in danger of injury when the knife enters the anterior chamber; furthermore prolapse of the iris is less likely to occur than if the incision was placed further back. As the aim of the incision is to make a linear opening into the angle of the anterior chamber the whole thickness of the sclera has to be traversed throughout the entire $5 \mathrm{~mm}$., hence it would be impossible to complete it with one stroke of the knife without sudden entry into the anterior chamber with consequent injury to the iris and particularly the lens, therefore, the incision is made with a series of cuts atl in the same place and all of the same length, until the anterior chamber is entered, this usually taking place by a small opening in the centre of the wound. The cutting edge of the knife should always be perpendicular to the antero-posterior curve of the sclera to avoid making a sloping incision with bevelled edges which tends to separate the superficial scleral fibres of the anterior portion of the wound which would make for difficulty in completing the inner portion of the incision.

The anterior chamber now having been entered at a small point in the centre of the wound, there is a slow leakage of aqueous from it and therefore slow decompression is achieved. As soon as the anterior chamber has been decompressed, the point of the knife is inserted into the small opening with the cutting edge directed upwards and the inner portion of the incision completed medially and laterally.

It is during the decompression of the anterior chamber and the subsequent enlargement of the inner portion of the incision that the complication of prolapse of the iris may occur; but providing the knife is sharp and little pressure has been used in making the cuts, the use of the scleral hook for fixation to avoid further pressure on the globe and the sphincter pupillae is well under the influence of the previously administered miotic, this complication seldom arises. If it does occur the iris can usually be reposited and this should be done before continuing. Very occasionally the iris will not remain inside the anterior chamber after many repositings; in this event a peripheral iridectomy will prevent the iris from making further appearances into the lips of the wound; however, as this complication will not arise if the above precautions are taken, one of the previously mentioned main points in favour of this operation will not have been missed.

Fixation still being maintained with the scleral hook in situ, but now held by the assistant, the operation is continued by making two forward cuts in the sclero-corneal tissue; these are made with small 
sharp pointed straight or curved scissors. Each of these forward cuts commences at the extremities of the original incision making an angle with it of approximately 45 degrees and extending forwards for $2 \mathrm{~mm}$., that is, $1 \mathrm{~mm}$. of sclera and $1 \mathrm{~mm}$. of cornea compose each cut. A triangle comprised of the two tissues is thus formed with a $4 \mathrm{~mm}$. base of scleral tissue and two sides each $2 \mathrm{~mm}$. in length containing equal portions of sclera and cornea. The distal ends of these two forward cuts do not completely meet so that the apex of the triangle is not completed and a small portion of corneal tissue is left in continuity with the rest of the cornea, resulting in mobility of the triangle only in a vertical direction.

Just prior to commencing these forward cuts the tip of an iris repositor is kept between the lips of the base incision by the free hand of the operator until the cuts are completed in order :

(a) to separate the lips of the incision so that the point of the scissors may be introduced into the wound without difficulty when commencing and completing each forward cut,

(b) to keep the iris away from the point of the scissors,

(c) to ensure against the iris prolapsing at this stage.

In those cases where there are peripheral anterior synechiae at the apex of the triangle these adhesions may sometimes be broken down with a repositor; at other times the adhesions may require division with scissors, thereby freeing the iris from the posterior surface of the cornea. A repositor should then be passed into the anterior chamber to ensure that free communication has been established between it and the triangular opening in its angle. The operation is completed by removing the scleral hook and repositing the conjunctival flap under the upper lid. No sutures are inserted into this tissue so that no tension is exerted upon it with subsequent pressure and closure of the triangular valve beneath it.

The immediate instillation of eserine $\frac{1}{2}$ per cent. in both acute and chronic cases prior to bandaging the eyes, is in the opinion of the writer a safeguard against prolapse of the iris during the 24 hours before the first dressing when the anterior chamber is attempting to reform. Atropine sulphate 1 per cent. should be instilled at the first dressing and at successive 24 hourly dressings until digital massage of the globe is commenced; the use of atropine here being solely to raise the intra-ocular pressure behind the mobile triangular flap of sclero-corneal tissue, thereby helping to keep it open.

The time to institute gentle digital massage being when the conjunctival flap has completely sealed off and there is no leakage of aqueous into the conjunctival sac; this may be ascertained by the daily instillation of fluorescein. If massage is employed before, there may be a sudden rush of aqueous through the permanent 
incisions taking with it the iris and probably necessitating further surgical interference in the form of an iridectomy. On the other hand once the conjunctival flap has healed, there is sufficient back pressure on the aqueous in the anterior chamber to prevent its sudden escape through the triangular opening, there is now no danger of the iris prolapsing.

As soon as massage has been started it may be carried out once or twice daily during the post-operative period to ensure the passage of aqueous through the opening and to prevent healing occurring at its edges. The patient is allowed up at this juncture and can usually be discharged around the tenth day. He is instructed to massage the eyeball gently daily through the lower lid and is seen at weekly. intervals until it has become obvious that a permanent filtering cicatrix has been produced; at this stage massage is discontinued.

\title{
OPHTHALMO-NEUROLOGICAL SYMPTOMS IN CONNECTION WITH MALIGNANT NASOPHARYNGEAL TUMOURS*
}

BY

\author{
DR. ERIK GODTFREDSEN
}

COPENHAGEN

\begin{abstract}
From Radiumhemmet, Stockholm (Professor L. Berven), Kung Gustaf $V^{\prime}$ tes Jubilaumsklinik, Lund (Professor L. Edling), and the Radiological Clinics of Copenhagen (Physician-in-Chief, Jans Nielsen) and Aarhus (Professor C. Krebs).
\end{abstract}

Mr. Chairman, Ladies and Gentlemen,

I feel greatly pleased and honoured to be allowed to speak before this dignified audience and thus to re-establish the connection between British and Danish ophthalmology, a connection which was previously so intimate and profitable. Danish ophthalmologists, among whom the best known are Bjerrum, Tscherning, Edmund Jensen, Heerfordt, Lundsgaard, and Roenne, are greatly indebted to British ophthalmologists, whose best known representatives with us are men like Young, Mackenzie, Bowman, Priestley-Smith, Marcus Gunn and Duke-Elder.

During the gloomy years of German occupation Danish ophthalmology greatly missed the inspiration from British ophthalmology. Now that I stand here as the first Danish speaker after the war I

* Read before the Ophthalmic Section, Royal Society of Medicine, London, November 14, 1946. + Teceived for publication, December 6, 1946. 